\title{
ACERCAMIENTO AL ESTUDIO DE LAS URBANIZACIONES CERRADAS EN COSTA RICA: NOTAS METODOLÓGICAS
}

\section{APPROACH TO THE STUDY OF GATED COMMUNITIES IN COSTA RICA: METHODOLOGICAL NOTES}

\author{
Alejandro Alvarado Alcázar* \\ Gustavo Jiménez Barboza**
}

Lo de afuera y lo de adentro son, los dos, íntimos, están prontos a invertirse, a trocar su hostilidad. Si hay una superficie limite entre tal adentro y afuera, dicha superficie es dolorosa en ambos lados. La poética del espacio. Gastón Bachelard

RESUMEN

El presente artículo plantea algunos aspectos a considerar en el estudio de las urbanizaciones cerradas y en especial, las dinámicas que se dan al interior de estas para el caso costarricense. En primer lugar, se muestran las condicionantes surgidas en el contexto de la sociedad neoliberal y la relación que tiene con la formación del miedo en el sujeto, así como, la conexión que posee con la proliferación de urbanizaciones cerradas en la región y en el país. Seguidamente, se examinan las principales formas en que se ha abordado el estudio de esta forma urbana en la región.

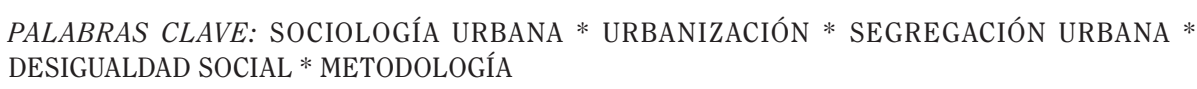

\section{ABSTRACT}

This article presents some aspects to be considered when studying gated communities and the dynamics found within these communities for the Costa Rican scenario. First, we show the conditions arising in the context of neoliberal society and the relationship that it has

\footnotetext{
* Instituto de Investigaciones Sociales (IIS) de la Universidad de Costa Rica (UCR). al.alcazar@hotmail.com

** Consultor independiente en temas de Planificación Urbana y Ordenamiento Territorial. gustjim@gmail.com
} 
with the formation of fear in the subject, as well as its connection with the proliferation of gated communities in the region and the country. Then, we examine the main approaches the study of this urban form has had in the region.

KEYWORDS: URBAN SOCIOLOGY * URBANIZATION * URBAN SEGREGATION * SOCIAL INEQUALITY * METHODOLOGY

\section{INTRODUCCIÓN}

El presente artículo examina los desafíos metodológicos que supone el estudio de las urbanizaciones cerradas en Costa Rica, desde la objetivación de la situación de entrevista planteada por Pinçon y Pinçon (2012), en la que el sociólogo ocupa una "posición dominada"1 frente a su sujeto/objeto de estudio. Al tratarse de un tipo de investigación sociológica cuyo sujeto/objeto pertenece principalmente, a las clases medias altas y en algunos casos, a las clases altas, se establece un tipo de relaciones entre las partes, bajo la cual las condicionantes generadas en esta situación en particular, colocan al sociólogo en una posición de "subordinación", diferenciando otro tipo de escenarios en donde esta posición no se da. Dicho esto, en primer lugar, se mostrarán las condicionantes surgidas en el contexto de la sociedad neoliberal y la relación que tiene con la formación del miedo en el sujeto, así como, la conexión que esto guarda con la proliferación de urbanizaciones cerradas. Seguidamente, se repasan algunas de las investigaciones generadas, principalmente en América Latina, sobre esta forma urbana emergente, poniendo énfasis en las limitaciones expuestas por las y los investigadores en relación con la aproximación al campo y sobre todo, en la relación cara a cara con las y los miembros de las clases medias altas y altas. Por último, se analiza dicha situación para el caso particular de Costa Rica, desde la experiencia generada en la investigación en curso "Urbanizaciones Cerradas en Costa Rica: transformaciones so-

1 El concepto de posición dominada en la investigación refiere a una valoración de capitales en el sentido propuesto por Bourdieu y abordado por los autores, más adelante se ampliará la discusión respecto a este tema. cio-espaciales, nueva urbanidad y segregación socio-residencial en el distrito de San Rafael de Escazú (1990-2010)".

\section{DEL MIEDO Y OTROS DEMONIOS}

La proliferación de las urbanizaciones cerradas como forma urbana masiva se sitúa en el contexto de los años 80. Dicha afirmación consta no solo para los países de la región, sino para otras regiones donde esta forma urbana se ha ido expandiendo de manera acelerada, hasta constituirse en la solución residencial preferida entre las clases medias $y$ altas.

Esta década es el decenio del despegue de la nueva fase del sistema capitalista: el neoliberalismo. Este modelo se cimienta como el modelo hegemónico de un nuevo escenario político, económico y cultural, codificado bajo el signo de la supremacía del mercado y sus reglas de juego, las cuales deben ser liberadas de las ataduras que supone la presencia del Estado, para allanar el camino a la acción privada.

Para Bellet (2007), las urbanizaciones cerradas son un producto neoliberal en el que se instala la idea de que el mercado es el único con capacidad para gestionar con eficiencia - mediante la privatización - la demanda por una solución residencial por parte de las clases medias $y$ altas.

En este sentido, siguiendo a Harvey (2007), se puede enmarcar este fenómeno como parte de las soluciones espaciales operadas por el capital, con el fin de superar sus crisis cíclicas producidas por su propia lógica interna. El capital especulativo, entre estos el inmobiliario, que predomina en la fase actual del sistema capitalista, recurre a la organización del espacio como forma de circular el capital, en un período de estancamiento de la economía. Según 
este autor, el sistema capitalista: “...produce un paisaje geográfico adecuado a su propia dinámica de acumulación en un momento particular de su historia, sólo para tener que destruir y reconstruir ese paisaje $y$ adaptarlo a la acumulación en una fecha posterior" (ibid.: 77).

El repliegue del sujeto, su sustracción desde lo público hacia lo privado, con la esperada reclusión de los principales ámbitos de reproducción de la vida cotidiana producida por una realidad que se le presenta y representa como agresora, es un fenómeno que está en la base de la huida de estas clases a espacios donde la presencia de dispositivos de seguridad $y$ vigilancia generan la sensación de protección demandada (Davis, 2003).

El malestar se traduce en miedo y el miedo se traduce en encierro, delineando una morfología urbana basada en una cartografía social estructurada bajo una lógica de "ganadores" y "perdedores" (Svampa, 2008). En este sentido, la ciudad se insulariza, es decir, se producen islas de riqueza e islas de pobreza, aparentemente desconectadas entre sí pero que en el fondo, son las dos caras de la profunda fractura social generada por la nueva realidad $y$ cuyas conexiones tienen un profundo sustrato de clase, en donde el desarrollo geográfico desigual se torna paroxístico (Harvey, 2007).

Bajo esta retórica del miedo, se estructura un binarismo jerárquico (Soja, 2008); es decir, un ordenamiento territorial por categorías, bajo el cual el par yo/otro se asume en confrontación, una tensión constante que enfrenta a los sujetos en el espacio bajo una amenaza permanente de agresión, real o ficticia, resultante en una geografía del miedo, como señala el propio Soja, el zeitgeist y el raumgeist ${ }^{2}$, el espíritu temporal y espacial de la época, donde está marcado por la intensificación del control social y espacial (ibid.: 425).

En palabras de Davis, se trata de la compartimentación de la vida pública, con lo cual se genera a la vez, la destrucción del espacio público (2003: 197), se produce así una doble

2 Raumgeist, del alemán raum=espacio $y$ geist=espíritu, es un concepto utilizado por Soja (2008) para designar la expresión espacial del espíritu de los tiempos. crisis: de integración y de identificación (Giglia, 2003). La primera sería el resultado de las condiciones de creciente desigualdad social sumada a los procesos de exclusión social de sectores cada vez más amplios de la sociedad (ibid.: 5). Por su parte, la segunda sería el resultado de reconocerse en la ciudad, o sea, de identificarse con ella como conjunto, con lo cual "...la crisis del espacio público no es solo una crisis de la forma urbis, sino que es al mismo tiempo crisis de la urbanidad como arte de vivir juntos..." (ibid.: 5).

Tal como fue mencionado, las urbanizaciones cerradas se han generalizado en todo el mundo; sin embargo, en nuestra región, la más desigual de todas, la expansión de estas no tiene comparación con ninguna otra. Si bien, los años 80 marcan el inicio de un nuevo urbanismo cuyo rasgo central es el confinamiento en espacios cerrados de los principales ámbitos de reproducción de la vida cotidiana — residencia, trabajo, estudio, consumo-, es durante los años 90 , período en el cual el proceso de desmantelamiento de los estados de la región toma más fuerza, cuando esta nueva forma urbana se torna masiva (Janoschka, 2002).

Este anti-urbanismo de ascendencia norteamericana, desplaza a la ciudad-centro, inspirada por Europa, como modelo de organización de la vida urbana. Bajo esta, toda la vida social de una nación y por lo tanto, todo "lo moderno", se escenificaba en la ciudad: la economía, la política y la cultura eran ámbitos netamente urbanos. Sin embargo, con el nuevo modelo norteamericano, la suburbanización se instala como la nueva realidad de las ciudades de la región, marcada por el éxodo desde el centro de la ciudad hacia puntos ubicados en la periferia (Sabatini, 2006).

Con esto se altera, el patrón de segregación tradicional, pasando de uno que semejaba el observable en las ciudades europeas a uno similar al que se observa en las ciudades norteamericanas, donde las distintas áreas de la ciudad son marcadamente homogéneas (Sabatini, 2006). En el primero, si bien, las elites se concentraban en torno al centro, con acceso a la mejor infraestructura, mientras un polo de ciudad moderna se desarrollaba en 
una dirección geográfica de la periferia, donde se ubicaban sectores medios $y$ altos, $y$ una periferia lejana alojaba a las clases populares, era posible notar una significativa diversidad social $^{3}$ a lo interno de los distintos puntos de la ciudad.

En el caso de Costa Rica, este proceso se escenifica, aunque con ritmos propios, debido al carácter sui generis de aplicación del modelo neoliberal (Hidalgo, 2000). La crisis del modelo de acumulación y crecimiento gestada durante los años 80 , genera nuevas formas urbanas (Fumero, 2009), en las cuales el capital privado se constituye como actor central en la política urbana de las últimas tres décadas, definiendo el uso de los suelos y el valor de la tierra (ibid.: 8-9).

Este proceso asume su forma más acabada en las direcciones este y oeste del Gran Área Metropolitana, en particular Escazú. Diseñado como ciudad de portada, este cantón es la imagen del llamado proceso de gentrificación (Marcuse, 1997), en el cual un sector de la ciudad se "desconecta", operando como realidad propia, ajena a la realidad que transcurre en el conjunto, tal como lo manifiesta Arrieta (1998), al señalar que el proceso de ordenamiento territorial del cantón se llevó a cabo por la acción de los grupos organizados, con lo cual este se desarrolló de acuerdo a los intereses locales, sin referencia a intereses regionales, ni nacionales. Pero este proceso no transcurre de manera uniforme en toda la ciudad, pues este tipo de desarrollo se centra en el distrito de San Rafael, donde se agrupa la mayor parte de los emprendimientos comerciales y residenciales que caracterizan a la nueva urbanidad (Bellet, 2007).

\section{EL ESTUDIO DE LAS URBANIZACIONES CERRADAS EN LATINOAMÉRICA: DIFICULTADES METODOLÓGICAS}

Como se señaló en la sección anterior, el fenómeno de las urbanizaciones cerradas en Costa Rica, comparte un conjunto de rasgos con lo ocurrido en otros países de la región

3 Es importante señalar que esta significativa diversidad social refiere a la coincidencia espacio-temporal de diferentes grupos de la escala social (altos, medios y bajos), con la excepción de los sectores calificados como marginales. Ver Sabatini, 2006: 4. latinoamericana, pese a las particularidades de la implementación del modelo neoliberal en el país. Entre los rasgos similares que se presentan se pueden citar: son principalmente los sectores de clases medias $y$ medias altas los que recurren a este tipo de forma residencial, y el desarrollo de estos ocurre principalmente hacia la periferia de las grandes conurbaciones, pero sin desligarse de estas mediante la conexión con los sistemas de carreteras y los causantes de la "elección" de una urbanización cerrada, como el miedo, la (in)seguridad, el estilo de vida, etc.

Para el caso latinoamericano, el estudio de las urbanizaciones cerradas tomó relevancia a principios de los años 90 , periodo en que se potenció la construcción de este tipo de forma urbana por parte de los actores inmobiliarios privados, en consonancia con el crecimiento de la nueva oferta inmobiliaria promovida por el modelo neoliberal en la región y establecida como solución residencial para nuevos sectores de las clases medias y medias altas ${ }^{4}$. Como lo señala Svampa (2004), son los sectores medios los que empiezan a habitar estos lugares, ya que los sectores altos desde antes tenían la capacidad de acceder a esta clase de encerramiento (ibid.: 44).

Todas estas formas residenciales lo que mantienen en común es "... el cercamiento perimetral y la seguridad privada" (ibid.: 23). Para casos como los de Argentina o Brasil, desde los años 20 y 30 existían countries para las burguesías nacionales, quienes utilizaban estos lugares como segundas casas. De los años 80 en adelante, con el aumento en la brecha social, estos countries se transforman paulatinamente en residencias permanentes, en consonancia con la construcción de un imaginario que concibe la ciudad-centro como un lugar peligroso, mientras que las localizaciones en la periferia del casco urbano, se constituyen como las "mejores opciones" para escapar de la creciente inseguridad, tanto real como imaginada.

$4 \quad$ Para el caso costarricense es claro que desde mediados de los años 80, con los Programas de Ajuste Estructural se comienza a dar el acceso de nuevas clases medias ligadas principalmente, al sector servicios $y$ un descenso de aquellas que estaban ligadas a las dinámicas económicas del modelo anterior (Cordero, 2005). 
La mayoría de estudios sobre urbanizaciones cerradas se han concentrado en un examen crítico basado en el marco histórico-social que las produce, aunque con escasa presencia in situ, con excepción de algunos trabajos (Svampa, 2004 y 2008; Low ${ }^{5}, 2004$ y Thuillier, 2005). Esto se debe, en parte, a que el ingreso es sumamente controlado, por lo que el trabajo de campo y la recolección de información de fuentes primarias están limitados por las posibilidades de contacto en el interior de estas.

En muchos de los trabajos consultados, se observa que la mayor cantidad de entrevistas hechas son a personas que residen en los alrededores de estas urbanizaciones, personas que trabajan en estas, funcionarios de gobiernos locales o desarrolladores inmobiliarios (Girola, 2005; Roitman, 2004; Ueda, 2004 y Vidal-Koppmann, 2007), exclusivamente para el análisis de la morfología urbana que estas modifican (Arizaga, 2000; Velázquez, García, Borges y García, 2003; Moreno, 2009) o midiendo el nivel de segregación que estas generan según mediciones a partir de Necesidades Básicas Insatisfechas (Pujol, Sánchez y Pérez, 2011).

En resumen, los trabajos producidos en América Latina se han enfocado principalmente, en comprender el "afuera" de las urbanizaciones cerradas, su relación con la ciudad "abierta" y los procesos económicos que se generan alrededor (centros comerciales, gimnasios, supermercados, entre otros.). Por el contrario, pocos trabajos en la región se han ocupado de describir la dinámica interna que se da en estas el "adentro". Esto supone una limitación teórico-metodológica para estudiar la relación afuera-adentro, debido a los escasos datos que se manejan del "adentro" en comparación con los del "afuera".

$5 \quad$ En el caso del trabajo de Low - que se concentra en las manifestaciones de este fenómeno en Norteamérica - se da una posición distinta del investigador, ya que forma parte de las clases sociales que tienen acceso a este tipo de urbanizaciones en los Estados Unidos, así como tiene familiares y conocidos en este tipo de urbanizaciones, lo que le permite realizar etnografía durante seis años, teniendo una cercanía empírica mayor con el sujeto/objeto de estudio.
LA POSICIÓN SUBORDINADA: ESTUDIANDO LAS URBANIZACIONES CERRADAS EN COSTA RICA

El trabajo de campo en las urbanizaciones cerradas en el área de San Rafael de Escazú, durante el año 2012, fue el punto de partida para el análisis sobre el cómo se están estudiando las urbanizaciones cerradas en el país, a partir de los planteamientos sobre la entrada al campo analizados por Pinçon y Pinçon (2012) y Bourdieu (2003).

Desde el análisis de Bourdieu, efectuar la "objetivación participante", es decir, “... la objetivación del sujeto de la objetivación, del sujeto analizante, en resumen, del investigador mismo" (p. 87), es una condición necesaria para comprenderse como investigador dentro de un contexto determinado de diferencias de capitales. En este sentido, como punto de partida se reconoce que ninguno de los dos investigadores que participan del proceso de investigación reside en una urbanización cerrada ni tampoco en el sector de Escazú, en otras palabras, se es ajeno tanto a esta forma urbana como al contexto en el que se encuentra, desde un punto de vista vivencial.

No se puede negar la procedencia del investigador en el análisis de un objeto de estudio, se debe realizar una retrospectiva que permita ver los orígenes sociales, económicos y culturales que se tengan, así como realizar un análisis crítico de nuestro inconsciente histórico, entendiendo este inconsciente como el conjunto de conocimientos que son producto de nuestra formación académica (ibid.: 91). Este análisis lleva al resultado de que como investigadores y como personas dentro de un estructura social somos un "otro" para las personas que viven dentro de estas urbanizaciones cerradas, motivo por el cual hay que generar una estrategia específica para lograr la obtención de datos de dichas personas, realizando en este proceso una vigilancia epistemológica constante $y$ una suerte de red de contactos estratégicos que permitan acceder a estas personas. Esta "otredad" en relación con el objeto de estudio, está presente en todas las investigaciones en ciencias sociales; sin embargo, para el caso de clases medias y medias altas, lo mismo que para las clases altas, la posesión de determinados tipos de capital —en el sentido 
planteado por Bourdieu-supone una relación diferenciada con el investigador, en comparación, por ejemplo, con lo que sucede con las clases bajas.

Es importante mencionar que las barreras físicas actúan también como barreras simbólicas, en tanto fragmentan el espacio urbano percibido por los residentes de estas formas residenciales, como por las personas que viven y transitan en los alrededores de estas. Además, hay una mediación en la interacción, en tanto hay que realizar todo un ritual para ingresar a estas: inspección en la entrada por parte de los guardias de seguridad, preguntas constantes sobre las razones de estar ahí, restricción para la toma de fotografías, ser recibido por una empleada doméstica o un intercomunicador y finalmente, con los dueños de la casa. Esto difiere un poco de la forma más directa que se tiene con las personas que viven en los lugares aledaños, en donde basta con tocar una puerta o un timbre para conocer al dueño de la casa. Esta situación para acceder con los residentes de las urbanizaciones cerradas dificulta el trabajo de campo, pero a la vez demuestra las diferentes formas de relacionamiento social que se pueden o no tener con las personas en el espacio urbano, en este sentido, diferenciado según la capacidad de aislarse de la ciudad abierta.

Pinçon y Pinçon muestran como en la situación de encontrarse en una posición de sujetos con un "otro", lo que se está dando es un proceso de habitus confrontados (2012: 340), es decir, formas de vida $y$ relacionamiento social distintos. En la mayoría de investigaciones en ciencias sociales se trabaja con sectores de clases populares o clases medias, lo cual produce, que el investigador ocupe una posición "dominante" por la relación particular entre los habitus, es decir, que logre mantener bajo control la dinámica de recolección de datos.

Esta situación se da de manera distinta en el caso de las clases medias altas, con respecto a los sectores de otro estrato o clase social, aunque la persona que es entrevistada no necesariamente quiera poner en evidencia su distinción social, simbólicamente sus bienes la demuestran. Como lo mencionan Pinçon y Pinçon:
La entrevista se desarrolla en el domicilio, las condiciones están reunidas para que la persona interrogada sea conducida a poner en evidencia, incluso contra su voluntad, los capitales que tiene. El sociólogo se encuentra en una posición dominada a pesar de que el entrevistado no tiene la intención, de manera deliberada, de ejercer ninguna relación de dominación (2012: 343).

Para el caso costarricense, lo que se da en el momento de ingresar a una vivienda con una serie de bienes particulares, es que quedan en evidencia las desigualdades de capitales, tanto en términos de capital económico, como en capital social y cultural. Se muestran bienes, conocimientos y relaciones sociales específicas a los cuales, solo personas de clases altas o medias altas pueden acceder, creando al instante la situación de "subordinación" para el investigador.

En el encuentro "cara a cara" se reducen muchos de los prejuicios que se puedan tener de las personas que viven en estas urbanizaciones, mas no se puede borrar la distancia objetiva que existe entre una parte y la otra (ibid.: 345). Ejemplo de esto es una entrevista realiza$\mathrm{da}$, en donde antes de ingresar a la casa, se nota una arquitectura colonial, las paredes acompañadas de enredaderas que se acoplan formando una suerte de imbricación entre lo natural y lo artificial, a lo interno se encuentran muebles minimalistas que denotan su alto costo económico, autos de lujo, así como, un patio interno en el centro de la casa con una fuente de características neoclásicas, acompañado esto de una ornamentación ideada especialmente, para que a partir de enredaderas se logre un efecto eco-urbano. Esta descripción simple del lugar, demuestra que se está ingresando a un espacio físico muy distinto al de una familia de clase media baja o clase baja, ya que prácticamente solo estos sectores tienen acceso a determinados bienes y una arquitectura de "lujo".

Una experiencia vivida en el trabajo de campo y que es claramente expuesta por Pinçon y Pinçon (2012: 359), es el tema del momento in situ de la entrevista, momento de la realización de preguntas clave que permitan 
responder a las dimensiones de análisis de la investigación, ya que en una experiencia tradicional en donde el sociólogo es el que maneja la entrevista, se da una situación de pregunta por parte del investigador $y$ respuesta por parte del investigado, sin embargo, al haber una posición de subordinación del sociólogo en estas entrevistas, muchas veces el entrevistador se convierte en entrevistado, sobre todo los casos en que los sujetos entrevistados poseen un amplio capital cultural. Esta situación provoca que la entrevista se desvié un poco de su estructura inicial, aunque este desvío también es aprovechable para la labor de la recolección de datos.

Lo principal al realizar una investigación de esta naturaleza es que como investigador o investigadores se puede tener una posición de subordinación, lo cual no es necesariamente un punto negativo, sino que hay que saber jugar con esta posición para sacar el mayor beneficio al momento del trabajo de campo y así entender de mejor forma el fenómeno que se está analizando; es decir, hay que utilizar esta posición de sociólogo subordinado como herramienta epistemológica con el fin de mejorar el estudio de las élites.

\section{CONSIDERACIONES FINALES}

A partir de lo expuesto, se pueden generar algunas cuestiones importantes en el estudio de las urbanizaciones cerradas. La primera cuestión es que al entender que el contexto neoliberal no modifica únicamente lo económico, sino otras esferas de la vida social como lo cultural, lo político, lo social, entre otros, hay que tener claro su papel en la constitución del sujeto, tanto aquellos que viven dentro, como los que están afuera y en muchos casos desean estar adentro. Esto ayuda a comprender el por qué existe en muchas personas el deseo y/o la idea de necesidad de vivir segregado de un "otro" amenazante, una situación que puede en gran medida explicar la proliferación de las urbanizaciones cerradas en el país. Si bien es cierto, el miedo puede ser uno de los mayores motivadores para vivir en una de estas urbanizaciones, no hay que absolutizar la causalidad, ya que otras situaciones como el estatus o la distinción social, también son factores a considerar.
Es importante comprender este aspectos como una expresión de los sectores medios altos ascendentes de las sociedades latinoamericanas, esto relacionado a dos fenómenos: 1) el aumento en la capacidad adquisitiva de nuevos sectores vinculados a dinámicas económicas beneficiadas a partir de los 80 por el modelo económico neoliberal, lo que les permite tener capacidad de poder acceder a esta clase de urbanizaciones y 2) debido a las dinámicas económicas del modelo, hay un ensanchamiento de la brecha social $y$ esto genera una brecha urbana, en la cual se da una dinámica de segregación, tanto de los sectores subalternos, como de los sectores económicamente más ostentosos, los cuales tienen la capacidad de "huir" de los otros sectores a través de la auto-segregación.

Otro tema importante a resaltar es que dentro de esa dinámica, el investigador tiene que comprender en el contexto en que se ubican las urbanizaciones cerradas, es decir, realizar una auto-inspección que deje en claro desde cual historia de vida se estudia las urbanizaciones cerradas, ya sea como una persona de un mismo origen social o como un "otro" que intenta entender las lógicas que se dan en esta clase de desarrollos urbanos. La comprensión de esta situación da una importante clave metodológica y epistemológica para la comprensión de este objeto de estudio.

\section{BIBLIOGRAFÍA}

\section{LIBROS}

Davis, Mike. Ciudad de cuarzo. Madrid, España: Lengua de Trapo, 2003.

Giglia, Ángela. "Espacio público y espacios cerrados en la Ciudad de México". Espacio público y reconstrucción de ciudadanía. Kuri Ramírez (comp.). México: FLACSO, 2003.

Harvey, David. (2005). El "nuevo" imperialismo: acumulación por desposesión. Buenos Aires: Clacso, 2004.

Harvey, David. Espacios de esperanza. Madrid, España: Akal, 2007.

Low, Setha. Behind the gates. Life, security, and the pursuit of happiness in fortress America. New York, EEuU: Taylor \& Francis Books, 2004. 
Marcuse, Peter. "Walls of fear and walls of support". Architecture of Fear. Nan Ellin (ed.). New York, EEuU. Princeton Architectural Press, 1997: 101-114.

Soja, Edward. Postmetrópolis. Estudios críticos sobre las ciudades y las regiones. Madrid, España: Ediciones Traficantes de Sueños, 2008.

Smith, Neil. "El redimensionamiento de las ciudades: la globalización y el urbanismo neoliberal". Capital financiero, propiedad inmobiliaria y cultura, eds. Harvey D. y Smith N. Museo de Arte Contemporáneo de Barcelona (масва) - Universidad Autónoma de Barcelona (UAB). 2005: 59-78.

Svampa, Maristella. La brecha urbana. Buenos Aires, Argentina: Capital Intelectual, 2004.

Svampa, Maristella. Los que ganaron. La vida en los countries y barrios privados. Buenos Aires, Argentina: Biblos, 2008.

\section{REVISTAS}

Alvarado, Alejandro y Jiménez, Gustavo. "Urbanizaciones cerradas en Costa Rica: un nuevo objeto de estudio". Revista de Ciencias Sociales 137. San José, Costa Rica. Editorial de la Universidad de Costa Rica, 2012: 13-23.

Arizaga, Carmen. "Murallas y barrios cerrados, la morfología espacial del ajuste en Buenos Aires". Revista Nueva Sociedad 166, Venezuela. Nuso Editores, 2000: 22-32.

Arrieta, Omar. "Planes estratégicos, desarrollo urbano y ambiente. Descentralización y participación ciudadana en Escazú", Revista Ambien-tico 63. Heredia, Costa Rica. Escuela de Ciencias Ambientales de la Universidad Nacional de Costa Rica, 1998: 7-16.

Cordero, Allen. "Clases medias y movimientos sociales en Costa Rica". Revista de Ciencias Sociales 109-110. San José, Costa Rica. Editorial de la Universidad de Costa Rica, 2005: 157-166.

Franquesa, Jaume. "Vaciar y llenar, o la lógica espacial de la neoliberalización". Reis
118. España. Centro de Investigaciones Sociológicas (cIs), 2007: 123-150.

Fumero, Patricia. "La ciudad fragmentada: la Gran Área Metropolitana (GAM)". Revista Herencia 22(2). Costa Rica. Vicerrectoría de Acción Social de la Universidad de Costa Rica, 2009: 7-12.

Janoschka, Michael. "Discursos de inseguridad y la ciudad cerrada: mitos, realidades, barreras y fronteras de un producto inmobiliario "perfecto". Imaginales 2. México. Acción Social Universidad de Sonora, 2005: 11-35.

Pujol, Rosendo; Sánchez, Leonardo y Pérez, Eduardo. "La segregación social como determinante del desarrollo urbano. Barrios cerrados y autosegregación en las ciudades de San José y Heredia, Costa Rica”. Revista de Ciencias Económicas 1 (29). Costa Rica. Editorial de la Universidad de Costa Rica, 2011: 445-477.

Theodore, Nik; Peck, Jamie y Brenner, Neil. "Urbanismo neoliberal: la ciudad y el imperio de los mercados". Temas Sociales 66. Santiago de Chile. SUR Corporación de Estudios Sociales y Educación, 2009: 1-12.

Thuillier, Guy. "El impacto socio-espacial de las urbanizaciones cerradas: el caso de la Región Metropolitana de Buenos Aires". Revista Eure 93. Santiago de Chile. Instituto de Estudios Urbanos y Territoriales de la Pontificia Universidad Católica de Chile, 2005: 5-20.

\section{TEXTOS ELECTRÓNICOS}

Bellet, Carmen. "Los espacios residenciales de tipo privativo y la construcción de la nueva ciudad: visiones de Privatopía". Scripta Nova 245 (11). España. Universidad de Barcelona, 2007. En: $<$ http://www.ub.edu/geocrit/sn/sn-24508. htm $>$ [Recuperado el 03 de enero de 2013].

Bourdieu, Pierre. "La objetivación participante". Journal of the Royal Anthropological Institute 2 (9). Wiley, 2003. En: <https:// cined ocumentalyetnologia.files. 
wordpress.com/2013/09/pierre-bourdieula-objetivacic3b3n-participante.pdf> [Recuperado el 08 de abril de 2012].

Girola, María. "Nuevos paisajes residenciales en el Gran Buenos Aires: los emprendimientos cerrados entre el urbanismo escenográfico y el urbanismo afinitario". Revista Litorales 6. Argentina. Universidad de Buenos Aires (UBA), 2005. En: <http://dialnet.unirioja. es/servlet/articulo?codigo $=1309153>$ [Recuperado el 10 de marzo de 2013].

Grant, Jill y Mittelsteadt, Lindsey. "Types of gated communities". Environment and Planning B: Planning and Design 31. Lóndres, Inglaterra. Pion, 2004: 913-930. En: <http://architectureandplanning.dal. ca/planning/faculty/download/b3165. pdf> [Recuperado el 05 de abril de 2013].

Janoschka, Michael y Glazse, Georg. "Urbanizaciones cerradas: Un modelo analítico". Revista Ciudades 59. España. Instituto Universitario de Urbanística de la Universidad de Valladolid, 2003: 9-20. En: <http://michael-janoschka.de/ pdfs/Janoschka,\%20Michael\%20(2003)_ Urbanizaciones $\% 20$ cerradas_un $\% 20$ modelo\%20anal\%C3\%ADtico.pdf> [Recuperado el 13 de agosto de 2012].

Janoschka, Michael. "El nuevo modelo de la ciudad latinoamericana: fragmentación y privatización”. Revista EURE 85 (28). 2002: 11-29. En: <http://www.michaeljanoschka.de/pdfs/Janoschka,\%20 Michae1\%20(2006)_El_modelo_ de_ciudad_latinoamericana.pdf $>$ [Recuperado el 04 de noviembre de 2012]. Moreno, Ramón. "Inserción de los fraccionamientos cerrados en los espacios urbanos de Nogales". Topofilia: Revista de Arquitectura, Urbanismo y Ciencias Sociales 3 (1). México. Centro de Estudios de América del Norte (CEAN) de El Colegio de Sonora 2009. En: $<$ http://topofilia.net/coloquio09moreno. html> [Recuperado el 05 de abril de 2013].

Pinçon, Michel y Pinçon, Monique. "La entrevista y sus condiciones específicas".
Revista CS en Ciencias Sociales 9. Cali, Colombria. Facultad de Derecho y Ciencias Sociales Universidad Icesi. Enero-junio 2012: 335-336. En: <http:// www.icesi.edu.co/revistas/index.php/ revista_cs/article/view/1225/1676> [Recuperado el 04 de noviembre de 2012]. Roitman, Sonia. "Urbanizaciones cerradas: estado de la cuestión hoy y propuesta teórica". Revista de Geografía, Norte Grande 32. Chile. Pontificia Universidad Católica de Chile, diciembre 2004: 5-19. En: <http://www.redalyc.org/articulo. oa? $\mathrm{id}=30003201>$ [Recuperado el $13 \mathrm{de}$ agosto de 2012].

Sabatini, Francisco. La segregación social del espacio en las ciudades de América Latina. Banco Interamericano de Desarrollo, 2006. En: <http://www.iadb. org/intal/intalcdi/PE/2008/01437.pdf> [Recuperado el 23 de noviembre de 2012].

Ueda, Vanda. "Nuevas periferias y nuevas urbanizaciones: los condominios cerrados en la metrópoli de Porto Alegre, Brasil". vil Coloquio de Geografía Urbana. Barcelona, España. 2004. En: $<$ http://www.uib.es/ggu/pdf_viI\%20 COLOQUIO/22_UEDA_nuevasperiferias.pdf> [Recuperado el 25 de setiembre de 2012].

Velásquez, Carmen; García, Belén; Borges, Edith y García, Víctor. "La muralla urbana. Cambios de lenguajes urbanos y arquitectónicos". Territorios 9. Colombia. Universidad del Rosario, 2003: 75-88. En: <http://www.redalyc.org/ pdf/357/35700905.pdf> [Recuperado el 22 de julio de 2012].

TESIS

Hidalgo, Luis. "El cambio estructural del sistema socioeconómico costarricense desde una perspectiva compleja y evolutiva (1980-1998)". [Tesis para optar por el grado de Doctor en Economía Aplicada]. Universidad de Huelva, 2000.

Méndez, Jesús. "Las representaciones sociales de los habitantes de San Rafael de Escazú: su importancia en la determinación de los elementos 
constitutivos de la identidad de los grupos de altos ingresos económicos". [Tesis para optar por el grado de Licenciado en Antropología]. Universidad de Costa Rica, 2011.

Vidal-Koppmann, Sonia. "Transformaciones socioterritoriales de la región metropolitana de buenos aires en la última década del siglo xx. La incidencia de las urbanizaciones privadas en la fragmentación de la periferia”. [Tesis para optar por el grado de Doctorado en Ciencias Sociales]. Facultad Latinoamericana de Ciencias SocialesFLACSO, 2007. En: <http://flacsoandes. org/ dspace/handle/10469/1265\#.UqrtBfTuJvE > .

Fecha de ingreso: 13/01/2014 Fecha de aprobación: 05/09/2014 